\title{
The RNA Virome of Echinoderms
}

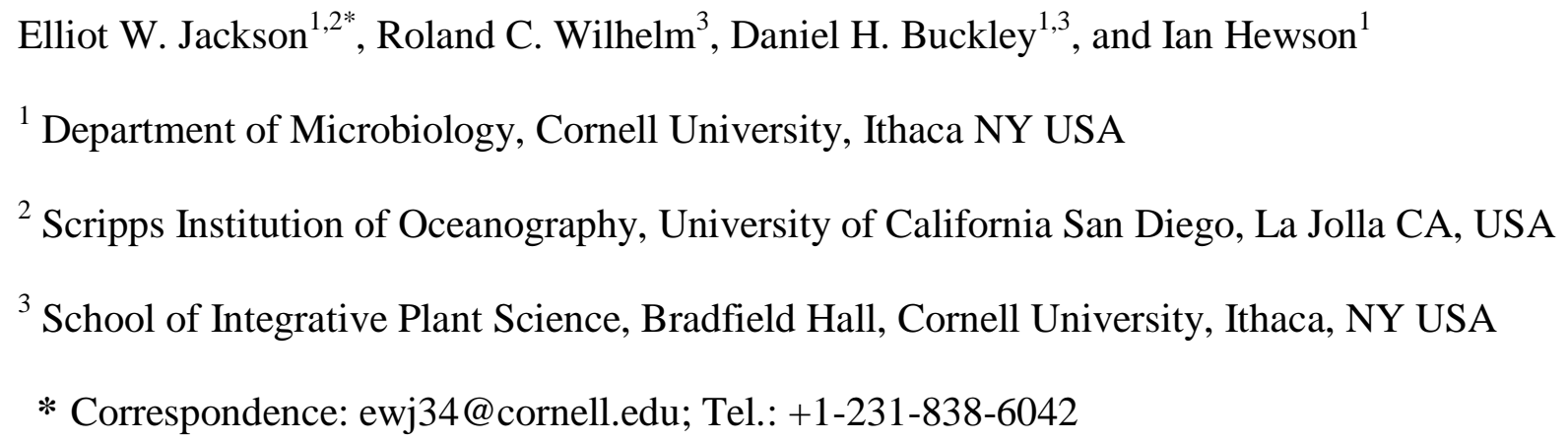

Key words: Viral discovery, Echinodermata, Invertebrates, RNA Viruses

\begin{abstract}
:
Echinoderms are a phylum of marine invertebrates that include model organisms,
\end{abstract} keystone species, and animals commercially harvested for seafood. Despite their scientific, ecological, and economic importance, there is little known about the diversity of RNA viruses that infect echinoderms compared to other invertebrates. We screened over 900 transcriptomes and viral metagenomes to characterize the RNA virome of 38 echinoderm species from all five classes (Crinoidea, Holothuroidea, Asteroidea, Ophiuroidea and Echinoidea). We identified 347 viral genome fragments that were classified to genera and families within nine viral orders Picornavirales, Durnavirales, Martellivirales, Nodamuvirales, Reovirales, Amarillovirales, Ghabrivirales, Mononegavirales, and Hepelivirales. We compared the relative viral representation across three life stages (embryo, larvae, adult) and characterized the gene content of contigs which encoded complete or near-complete genomes. The proportion of viral reads in a given transcriptome was not found to significantly differ between life stages though the majority of viral contigs were discovered from transcriptomes of adult tissue. This study illuminates the

27 biodiversity of RNA viruses from echinoderms, revealing the occurrence of viral groups in natural populations. 


\section{Introduction}

Metazoans harbor an enormous diversity and abundance of RNA viruses - a discovery

32 that has reshaped our understanding of viral evolution through expanded viral-host associations,

33 broadened phylogenetic diversity, and novel reconfigurations of genome architectures [1, 2].

34 Newly discovered viruses often blur the boundaries between well-known viral groups. For

35 example, prior to a recent expansion, the family Flaviviridae was typified by relatively uniform,

36 monopartite genomes, having a single 10-12 kb-long open reading frame (ORF), and being

37 vectored to mammals by arthropod. Metagenomics has led to the discovery of hundreds of novel

38 flavivirus genomes, redefining the genomic properties of this viral family, and extending their

39 host diversity beyond mammals [3-6]. To date, the exploration and systematization of

40 invertebrate RNA viruses have been skewed towards terrestrial arthropods, mainly insects,

41 leaving gaps in our understanding of the diversity, ecology, and evolution of RNA virus in other

42 invertebrate groups [1,7-12]. To help close this gap, we characterized the RNA virome of

43 Echinodermata - a phylum of marine invertebrates that are globally distributed throughout

44 Earth's oceans and represent an evolutionary crossroads in developmental biology as one of two

45 phyla that are invertebrate deuterostomes.

Wildlife disease and aquaculture are the two primary areas of concern regarding the

47 threat of viral outbreaks among echinoderms. Disease outbreaks of sea urchins and sea stars have

48 been documented at local, regional, and continental scales since 1898 and have gone unresolved

49 in regards to their etiology $[13,14]$. Certain species of sea urchins and sea cucumbers are valued

50 as seafood delicacies and the growing demands for these species in the seafood industry have led

51 to a rise in aquaculture farming $[15,16]$. Viral outbreaks pose a major concern for aquaculture

52 operations $[17,18]$, yet little is known about the identity, let alone virulence, of viruses that 
53 infect these animals [19-21]. Baseline knowledge of viruses present in wild populations can help

54 determine the etiology of future outbreaks and discern pathogenic versus non-pathogenic agents,

55 or those which may become more replicative under environmental stress. Thus, a census of viral

56 diversity will improve our future response when viruses impact the economic or ecological

57 function of echinoderms.

58 Parvoviruses - linear, single-stranded DNA viruses - are the best documented group

59 known to infect echinoderms since the discovery of a densovirus in a sea urchin metagenome

60 from Hawaii in 2014 [19]. Shortly after, another densovirus was found in various sea star species

61 and was implicated as the causative pathogen of the 2013/2014 Sea Star Wasting Syndrome

62 (SSWS) outbreak in the Northeast Pacific [22]. However, subsequent attempts to correlate

63 densoviruses to SSWS have not produced any clear association with pathology or disease [21-

64 26]. Regardless, this discovery prompted a series of investigations into the diversity, prevalence,

65 and association of these viruses with sea stars and SSWS [21, 24-26]. To date, no RNA virus

66 identified using -omic approaches has been proven to cause any pathology in echinoderms [21,

67 27]. However, one clear line of evidence that has emerged from the accumulation of -omic data

68 is that sea stars are infected by a diversity of viruses. We expect that they, and other

69 echinoderms, will be host to a novel, undocumented diversity of RNA viruses.

70 Sequencing-based 'viromics' approaches have been the primary method for the discovery

71 and characterization of echinoderm viruses. Other methods, like microscopy or culturing, are

72 laborious and low throughput or hindered by the lack of available cell lines from aquatic

73 invertebrates. All echinoderm virome studies, to date, have taken a viral metagenomic approach,

74 where shotgun metagenomics is performed on encapsidated nucleic acids that have been

75 enriched and selected for by chemical and/or nuclease treatment from size-filtered $(<0.2 \mathrm{um})$ 
76 tissue homogenates [19-22, 25-27]. Metatranscriptomes and transcriptomes are increasingly

77 used for viral discovery and have not yet been applied to echinoderms [28-31]. In the context of

78 a metazoan, a metatranscriptome refers to the sequencing of total RNA (with rRNA removed)

79 generally from samples pooled at the population level (multiple individuals of a species), while a

80 transcriptome is the sequencing of poly-A tailed mRNA (with rRNA removed) generally from an

81 individual organism [28, 32]. RNA-seq studies generating metatranscriptomes are typically for

82 the purpose of viral discovery as opposed to transcriptomes, which are created for the purpose of

83 analyzing gene expression patterns of an organism. In this study, we analyzed over 900 publicly

84 available transcriptomes from echinoderms to characterize the biodiversity and distribution of

85 RNA viruses. Together with previously published RNA viral metagenomes (i.e. a population of

86 genomes of RNA viruses), we conducted a systematic survey of RNA viruses associated with the

87 five major classes (Crinoidea, Holothuroidea, Asteroidea, Ophiuroidea and Echinoidea) of

88 Echinodermata.

\section{Methods}

89 The following sections detail the processing of the short-read libraries used for viral

90 discovery and the analyses performed on the viral sequences. The two sources of libraries were

91 transcriptomes and RNA-based viral metagenomes derived from various echinoderm species and

92 tissues. All libraries processed in this study were obtained from the NCBI's Short Read Archive

93 (Table S1) and the assemblies generated from this study and the database used for viral

94 discovery are accessible through the Open Science Foundation (https://osf.io/JXUAM).

95 Host transcriptomes and RNA-based viral metagenomes

A total of 903 paired-end transcriptomes derived from the five classes of echinoderm

98 hosts, including crinoid $(\mathrm{n}=18$; Crinoidea), sea cucumbers $(\mathrm{n}=178$; Holothuroidea), sea star ( $\mathrm{n}$

$99=179$; Asteroidea), brittle star $(\mathrm{n}=71$; Ophiuroidea $)$ and urchin $(\mathrm{n}=457$; Echinoidea $)$. Raw 
sequences were quality controlled using Trimmomatic [33], to clip adapters, and FastX [34], to

101 discard reads with lengths $<50 \mathrm{nt}$ and average quality scores $<30$. Transcriptomes were then

102 assembled using default parameters in Trinity (v2.1.1) [35]. Contigs less than $500 \mathrm{nt}$ were

103 discarded prior to viral annotation.

A total of 24 paired-end RNA viral metagenomes derived from sea cucumbers $(n=3$;

105 Holothuroidea) and sea stars (n=21; Asteroidea) were also retrieved from the Short Read Archive

106 (Table S1). Raw sequences were merged, trimmed to clip adapters and discard reads with

107 average quality scores < 20, and normalized to an average read depth of 100 using BBtools [36].

108 RNA viral metagenomes were assembled using Spades (v 3.11.1) with the -meta flag [37].

109 Contigs less than $500 \mathrm{nt}$ were discarded prior to viral annotation.

\section{Virus discovery and annotation}

We curated an RNA virus database for viral annotation. The database contained viral

113 amino acid sequences from Shi et al 2016, Wu et al 2020 and Wolf et al 2020, and from the

114 NCBI viral genome database after filtering for viral sequences from invertebrates and

115 invertebrates/vertebrates [1, 29, 38]. Duplicated amino acid sequences were removed from the

116 database using seqkit, yielding a total of 36,193 unique viral gene sequences. Echinoderm RNA

117 viruses were then identified by querying transcriptome/RNA viral metagenome assemblies

118 against the curated RNA viral database using DIAMOND BLASTx with the sensitivity 119 parameter adjusted to 'very-sensitive' and an e-value cutoff of $<10^{-20}$ [39]. Contigs with

120 significant similarity based on the BLAST criteria above were manually inspected in Geneious

121 Prime (v 2020.2.2), and queried against the NCBI non-redundant database using the default

122 BLASTp parameters to verify the viral annotation and assign putative gene function. BLASTp

123 results were also used to identify conserved protein domains. Genome illustrations were created 
124 by exporting the sequence viewer from Geneious Prime (v 2020.2.2) into Adobe Illustrator

125 (v25.4.1). Contigs with near identical matches to human and plant viruses and bacteriophage

126 were removed. ORFs containing an RdRP domain were used for taxonomic placement. If a

127 contig did not contain an RdRP sequence, a complete or partial ORF containing any conserved

128 protein domain was chosen. Contigs that did not contain a conserved protein domain were

129 removed from further analysis. Quality-filtered reads were mapped to viral contigs to obtain

130 relative abundance information using BBMap [40] using the 'semiperfect' flag which

131 accommodated ambiguous bases (with equivalent results achieved with the 'perfect' flag).

132

133

134

135

136

137

138

139

140

141

142

143

144

145

146

147

\section{Network analysis and phylogenetics}

The taxonomic relationships of all recovered viral sequences were first mapped using a network analysis. To place sequences into broad taxonomic groups, we downloaded amino acid sequences from the top NCBI BLASTp results for each of our recovered viral sequences. A network was built based on sequence similarity using the online EFI-EST portal with default settings (minimum length $=0$, maximum length $=50,000$, filter type $=$ e-value $\leq 10^{-5}[41]$. Nodes represent individual viral sequences and edges are the degree of similarity based off BLASTp pairwise similarity scores using a minimum pairwise similarity of $35 \%$. Clusters and singletons were removed from the network that did not contain any representative viral sequences with a RdRP sequence. The network was visualized in Cytoscape (v 3.8.2) using the 'organic' layout [42].

We further established the relatives of echinoderm RNA viruses based on RdRP phylogenies. Independent phylogenetic analyses were performed for viral orders using the type species designated by the International Committee on Taxonomy of Viruses. RdRP amino acid sequences were aligned using MAFFT [43] and phylogenies were inferred by a substitution 
148 model selected by smart model selection in PhyML 3.0 with branch support determined by

149 bootstrapping for 100 iterations [44]. The resulting phylogenetic tree was visualized and

150 annotated using FigTree v1.4.4 [45]. The Amarillovirales phylogeny was created from the

151 MAFFT alignment used in [46].

152 Results

153 We recovered a total of 347 viral contigs and 33 complete or near-complete genomes

154 from the 927 short read libraries analyzed. A total of 259 viral contigs were recovered from

155 transcriptomes, and 88 viral contigs from RNA viral metagenomes (Figure S1B). The mean viral

156 contig length recovered from RNA viral metagenomes (mean \pm standard deviation: 4,421 \pm 3077

$157 \mathrm{nt})$ was greater than from transcriptomes $(3,143 \pm 3102 \mathrm{nt}$; Figure S1A), and the size of

158 sequencing libraries was weakly correlated with viral read depth in transcriptome libraries $(\mathrm{p}=$

1590.002 , Pearson's $r=0.29)$ but not in RNA viral metagenomic libraries $(p=0.82, r=0.05$; Figure

160 S1C). On average the relative abundance of viral contigs as a proportion of total reads was low,

$161(0.0085 \%)$, ranging from $0.000023 \%$ to $0.29 \%(\mathrm{x} \square=0.0085 \%)$. The average percentage of viral

162 reads in viral metagenomes $(\mathrm{x} \square=0.39 \%)$ was $\sim 45$-fold higher than transcriptomes. However,

163 viral abundance was highly uneven in the viral metagenomes with the average dropping to

$1640.07 \%(\sim 8$-fold higher than transcriptomes) after excluding the top three most abundant samples.

165 Viral sequences were recovered from 111 of the 903 transcriptomic libraries and from all

166 five echinoderm classes (Figure 1). Sea cucumbers exhibited the highest prevalence of viral

167 contigs among all echinoderm libraries (i.e., individuals) screened, followed by sea urchins

$168(10 \%, 45 / 457)$, and the highest proportion among transcriptomes screened $(26 \%$; 47/178) (Figure

169 1A). The majority of viral contigs recovered from transcriptomes came from adult tissue (70\%)

170 compared to embryos (20\%) or larvae (10\%) (Figure 1B). Transcriptomes derived from

171 echinoderms during their larval stage had a slightly higher proportion of viral reads in their 
172 transcriptome $(\mathrm{x} \square=0.013 \%)$ than adults $(0.0095 \%)$, and more than embryos $(0.0034 \%$; Figure

$1731 \mathrm{C}$ ), but these differences were not significant (Kruskal-Wallis, chi-squared 1.65, p-value=0.80).

174 Over half of the viral contigs contained an RdRP sequence (186/347), with 96 of these

175 containing a complete or partial capsid sequence. Most viral contigs (215) contained at least a

176 partial capsid sequence, and 42 viral contigs contained another conserved viral domain such as a

177 methyltransferase or an RNA helicase domain (Supplemental Table 2). The majority of viral

178 contigs were taxonomically placed in the order Picornavirales $(n=235)$ (Figure 2$)$. The

179 recovered picornaviruses were distributed among a variety of families with the largest number

180 related to Marnaviridae, followed by Dicistroviridae, and Iflaviridae (Figure 3). Several unique

181 clades within Picornavirales were represented by complete or near-complete genomes and may

182 represent novel viral families (Figure 2). The second highest number of viral contigs were

183 recovered from Mononegavirales $(\mathrm{n}=12)$, with the remainder spread among seven orders $(\mathrm{n}=$

184 20): Durnavirales, Martellivirales, Nodamuvirales, Reovirales, Amarillovirales, Ghabrivirales,

185 and Hepelivirales. In general, the recovered viruses did not form new monophyletic clades

186 within Picornavirales (Figure 3) or Amarillovirales, Reovirales, and Mononegavirales (Figure

187 4). However, within the Hepivirales, the recovered viruses formed a distinct monophyletic clade

188 that is sister to the clade containing Orthohepivirus and Piscihepivirus.

$189 \quad$ Viral contig lengths ranged from $502 \mathrm{nt}$ to $12,989 \mathrm{nt}$. Complete and near complete

190 genomes were recovered from Picornavirales, Mononegavirales, Amarillovirales, and

191 Hepelivirales (Figure 5). The echinoderm picornavirus genomes exhibited three different open

192 reading frame arrangements, which spatially separated the genome by function according to

193 replication or encapsidation (Figure 3). The two most conserved protein domains related to

194 replication were the RdRP (pfam00680) and RNA helicase (pfam00910) domains with many of 
195 the genomes also containing BIR (pfam00653), DSRM (pfam00035), Sigma70 (pfam04539),

196 peptidases (pfam12381), and large tegument protein (PHA03247) domains. The conserved

197 capsid domains found among the picornavirus genome included: rhv-like (pfam00073), dicistro

198 VP4 (pfam11492), CRPV (pfam08762), and calici coat (pfam00915) domains (Figure 5). The

199 recovered hepeviruses and mononegaviruses contigs contained the expected replication proteins

200 but many lacked complete capsid proteins, indicating they were only near complete genomes

201 (Figure 5). The flavivirus genome previously discovered in sea cucumber [27] was completed

202 during our assembly, leading to the extension of a second complete ORF and extending the

203 genome size from 8,883 to $12,989 \mathrm{nt}$.

204 Discussion

205 The most prevalent RNA viruses in echinoderm transcriptomes and RNA viral

206 metagenomes were picornaviruses which are non-enveloped, single-stranded RNA (+ssRNA)

207 viruses. The order Picornavirales is comprised of eight families (Caliciviridae, Dicistroviridae,

208 Iflaviridae, Marnaviridae, Picornaviridae, Polycipiviridae, Secoviridae, and Solinviviridae) and

209103 genera and are among the most prevalent and diverse group of viruses found in -omics

210 surveys of animal and environmental samples [47, 29, 38, 48]. The majority of the echinoderm-

211 associated picornaviruses grouped into the Dicistroviridae, Iflaviridae, and Marnaviridae

212 families, but other recovered viruses also comprised novel clades (Figure 3) which supported our

213 expectation that the extant diversity of echinoderm RNA viruses is under sampled. The

214 Marnaviridae are known to be ocean virioplankton which infect single-celled eukaryotes, such

215 as phytoplankton and protists, but have also been found from metatranscriptomes from marine

216 bivalves [48, 49]. It is possible that the Marnaviridae we observed infect protists that are

217 symbionts or transiently associated with echinoderms. Alternatively, the host-range of the 
218 Marnaviridae family may extend beyond single-celled eukaryotes. The host range of many viral

219 groups has changed considerably in recent years, and there are examples of host ranges within

220 RNA viral families that do extend from protists to mammals, such as Reoviridae [50, 51]. All

221 classified species of Dicistroviridae and Iflaviridae infect arthropods, and have largely been

222 characterized due to the economic impacts of their pathogenicity though the host range of these

223 families likely extends far beyond arthropods given their presence in metatranscriptomes from

224 organisms in the phyla Mollusca, Cnidaria, and Platyhelminthes [31, 48, 52]. The disease

225 severity from infections of both families ranges from inapparent to lethal, supporting the

226 possibility that there may be non-pathogenic species infecting echinoderms.

228 associations, we observed Martellivirales, Nodamuvirales, Reovirales, Amarillovirales,

229 Mononegavirales, and Hepelivirales. Many of these echinoderm viruses phylogenetically cluster

230 with established invertebrate-infecting families or genera (i.e. Nymaviridae family within

231 Mononegavirales or Cardorevirus genus within Reovirales) while some represent evolutionary

232 novel lineages such as the flavivirus discovered from a sea cucumber (Figure 4C) [27]. Currently

233 it is unclear if members of the rare virosphere infect all classes of echinoderms or if some are

234 class specific. For example, the reovirus and flavivirus discovered in this study were only found

235 in sea stars and sea cucumbers, respectively, though we cannot rule out methodological biases

236 and insufficient sample size as proof of absence, requiring further research. Nevertheless, the

237 discovery of these viruses significantly expands the host range for many of these groups and

238 represents the first RNA viruses discovered from crinoids and brittle stars. 
241 53]. This pattern of abundance likely has a basis in biology, but in the case of our dataset, may be

242 inflated due to our use of transcriptomic data. The selection for polyadenylated transcripts during

243 RNA-seq library preparation biases towards +ssRNA viruses, like picornaviruses, which have

244 3'polyadenylated tails $[54,55]$. Studies utilizing a metatranscriptomic approach for RNA viral

245 discovery generally do not find such a highly skewed distribution towards +ssRNA but are

246 nevertheless the most abundant viral type [1, 29, 38, 48]. By utilizing viral RNA metagenomes

247 and transcriptomes we have uncovered the fullest diversity of RNA viruses associated with

248 echinoderms with the datasets available, though we expect future multi-omic efforts to reveal

249 additional diversity.

The greatest difference between the two -omic approaches used in this study for viral

251 discovery was the total number of viral contigs recovered and contig lengths. Viral RNA

252 metagenomes generally contained $>3$ viral contigs per library, which were $\sim 40 \%$ longer,

253 compared to transcriptomes, which contained 2.2 contigs per library. Additionally, the

254 proportion of viral reads recovered exhibited a weak correlation with library size for

255 transcriptomes but not for viral RNA metagenomes (Figure S1B). Thus, despite the efforts to

256 enrich for viruses in the viral RNA metagenomes, the majority of libraries had a similar percent

257 of viral reads compared to transcriptomes. Furthermore, these findings indicate that the efficacy

258 of recovering RNA virus improves with sequencing depth, likely due to the improved assembly

259 of sequenced found in low abundance.

260 The capacity for viral discovery using -omic approaches has greatly expanded our

261 understanding of biodiversity and host range, fundamentally shifting the perception of viruses as

262 solely pathogens to a more nuanced role as commensals or mutualists [1]. Performing a viral

263 census of hosts, like echinoderms, provides a useful context about the prevalence and association 
264 of viruses that can help understand future outbreaks or changes in the susceptibility of marine

265 animals due to stress from climate change and human activity. The full potential of -omic

266 approaches to understand the biological or ecological role of the diversity of viruses uncovered

267 will only be fully realized in partnership with advances in culturing techniques to study the

268 infection of naïve specimens [56, 57]. Our study provides a comprehensive survey of RNA

269 viruses present in echinoderm, contrasting the diversity and abundance of RNA viruses between

270 echinoderm classes and life stages. We hope this information provides valuable context for

271 advancing our understanding of the role of these viruses in marine hosts and ecosystems.

\section{Funding}

273 This work was supported by NSF grants OCE-1537111, OCE-1737127, and OCE-

2742049225 awarded to IH. This work was also supported by the Cornell Atkinson Center's

275 Sustainable Biodiversity Fund and Andrew W. Mellon Student Research Grant awarded to EWJ.

276 Author statements

277 The authors declare that there are no conflicts of interest. 


\section{References}

1. Shi M, Lin X-D, Tian J-H, Chen L-J, Chen X, et al. Redefining the invertebrate RNA virosphere. Nature 2016;540:539-543.

2. Shi M, Lin X-D, Chen X, Tian J-H, Chen L-J, et al. The evolutionary history of vertebrate RNA viruses. Nature 2018;556:197-202.

3. Bekal S, Domier LL, Gonfa B, McCoppin NK, Lambert KN, et al. A novel flavivirus in the soybean cyst nematode. Journal of General Virology 2014;95:1272-1280.

4. Fauver JR, Grubaugh ND, Krajacich BJ, Weger-Lucarelli J, Lakin SM, et al. West African Anopheles gambiae mosquitoes harbor a taxonomically diverse virome including new insect-specific flaviviruses, mononegaviruses, and totiviruses. Virology 2016;498:288-299.

5. Qin X-C, Shi M, Tian J-H, Lin X-D, Gao D-Y, et al. A tick-borne segmented RNA virus contains genome segments derived from unsegmented viral ancestors. Proceedings of the National Academy of Sciences 2014;111:6744-6749.

6. Shi $\mathbf{M}$, Lin X-D, Vasilakis $\mathbf{N}$, Tian J-H, Li C-X, et al. Divergent viruses discovered in arthropods and vertebrates revise the evolutionary history of the Flaviviridae and related viruses. Journal of Virology 2016;90:659-669.

7. Junglen S, Drosten C. Virus discovery and recent insights into virus diversity in arthropods. Current Opinion in Microbiology 2013;16:507-513.

8. Li C-X, Shi M, Tian J-H, Lin X-D, Kang Y-J, et al. Unprecedented genomic diversity of RNA viruses in arthropods reveals the ancestry of negative-sense RNA viruses. elife 2015;4:e05378.

9. Liu S, Chen Y, Bonning BC. RNA virus discovery in insects. Current Opinion in Insect Science 2015;8:54-61.

10. Remnant EJ, Shi M, Buchmann G, Blacquière $T$, Holmes EC, et al. A diverse range of novel RNA viruses in geographically distinct honey bee populations. Journal of virology 2017;91:e00158-17.

11. Käfer S, Paraskevopoulou S, Zirkel F, Wieseke N, Donath A, et al. Re-assessing the diversity of negative strand RNA viruses in insects. PLoS Pathogens 2019;15:e1008224. revealed by RNA-seq analysis: ecological and evolutionary implications. Msystems 2020;5:e00039-20. (editor). Developments in Aquaculture and Fisheries Science. Elsevier. pp. 179-186. 
14. Hewson I, Sullivan B, Jackson EW, Xu Q, Long H, et al. Perspective: Something Old, Something New? Review of Wasting and Other Mortality in Asteroidea (Echinodermata). Frontiers in Marine Science 2019;6:406.

15. Slater M. Use and Exploitation of Sea Cucumbers. In: Echinoderm Aquaculture. John Wiley \& Sons, Ltd. pp. 57-73.

16. Sun J, Chiang F-S. Use and Exploitation of Sea Urchins. In: Echinoderm Aquaculture. John Wiley \& Sons, Ltd. pp. 25-45.

17. Deng H, Zhou Z, Wang N, Liu C. The syndrome of sea cucumber (Apostichopus japonicus) infected by virus and bacteria. Virologica Sinica 2008;23:63-67.

18. Liu H, Zheng F, Sun X, Hong X, Dong S, et al. Identification of the pathogens associated with skin ulceration and peristome tumescence in cultured sea cucumbers Apostichopus japonicus (Selenka). Journal of Invertebrate Pathology 2010;105:236-242.

19. Gudenkauf BM, Eaglesham JB, Aragundi WM, Hewson I. Discovery of urchin-associated densoviruses (family Parvoviridae) in coastal waters of the Big Island, Hawaii. Journal of General Virology 2014;95:652-658.

20. Jackson EW, Bistolas KS, Button JB, Hewson I. Novel Circular Single-Stranded DNA Viruses among an Asteroid, Echinoid and Holothurian (Phylum: Echinodermata). PloS One 2016;11:e0166093.

21. Hewson I, Aquino CA, DeRito CM. Virome variation during sea star wasting disease progression in Pisaster ochraceus (Asteroidea, Echinodermata). Viruses 2020;12:1332.

22. Hewson I, Button JB, Gudenkauf BM, Miner B, Newton AL, et al. Densovirus associated with sea-star wasting disease and mass mortality. Proceedings of the National Academy of Sciences 2014;111:17278-17283.

23. Bucci C, Francoeur M, McGreal J, Smolowitz R, Zazueta-Novoa V, et al. Sea Star Wasting Disease in Asterias forbesi along the Atlantic Coast of North America. PLoS One 2017;12:e0188523.

24. Hewson I, Bistolas KS, Quijano Cardé EM, Button JB, Foster PJ, et al. Investigating the complex association between viral ecology, environment, and northeast Pacific sea star wasting. Frontiers in Marine Science 2018;5:77.

25. Jackson EW, Pepe-Ranney C, Johnson MR, Distel DL, Hewson I. A Highly Prevalent and Pervasive Densovirus Discovered among Sea Stars from the North American Atlantic Coast. Applied and Environmental Microbiology 2020;86:e02723-19. 
26. Jackson EW, Wilhelm RC, Johnson MR, Lutz HL, Danforth I, et al. Diversity of Sea StarAssociated Densoviruses and Transcribed Endogenous Viral Elements of Densovirus Origin. Journal of Virology 2020;95:e01594-20.

27. Hewson I, Johnson MR, Tibbetts IR. An unconventional flavivirus and other RNA viruses in the sea cucumber (Holothuroidea; Echinodermata) virome. Viruses 2020;12:1057.

28. Shi M, Zhang Y-Z, Holmes EC. Meta-transcriptomics and the evolutionary biology of RNA viruses. Virus Research 2018;243:83-90.

29. Wu H, Pang R, Cheng T, Xue L, Zeng $\mathbf{H}$, et al. Abundant and Diverse RNA viruses in insects revealed by RNA-seq analysis: ecological and evolutionary implications. Msystems;5.

30. Parry R, Asgari S. Discovery of novel crustacean and cephalopod flaviviruses: insights into the evolution and circulation of flaviviruses between marine invertebrate and vertebrate hosts. Journal of Virology 2019;93:e00432-19.

31. Cheng R-L, Li X-F, Zhang C-X. Novel Dicistroviruses in an Unexpected Wide Range of Invertebrates. Food and Environmental Virology 2021;1-9.

32. Zhang Y-Z, Chen Y-M, Wang W, Qin X-C, Holmes EC. Expanding the RNA virosphere by unbiased metagenomics. Annual Review of Virology 2019;6:119-139.

33. Bolger AM, Lohse $\mathbf{M}$, Usadel B. Trimmomatic: A flexible trimmer for Illumina sequence data. Bioinformatics 2014;30:2114-2120.

34. Gordon A, Hannon GJ. Fastx-toolkit. FASTQ/A short-reads preprocessing tools 2010;5:http://hannonlab.cshl.edu/fastx_toolkit.

35. Grabherr MG, Haas BJ, Yassour M, Levin JZ, Thompson DA, et al. Full-length transcriptome assembly from RNA-Seq data without a reference genome. Nature Biotechnology 2011;29:644-652.

36. Bushnell B. BBTools software package. URL http://sourceforge net/projects/bbmap 2014;578:579.

37. Bankevich A, Nurk S, Antipov D, Gurevich AA, Dvorkin M, et al. SPAdes: a new genome assembly algorithm and its applications to single-cell sequencing. Journal of computational biology 2012;19:455-477.

38. Wolf YI, Silas S, Wang Y, Wu S, Bocek M, et al. Doubling of the known set of RNA viruses by metagenomic analysis of an aquatic virome. Nature Microbiology 2020;5:1262-1270.

39. Buchfink B, Xie C, Huson DH. Fast and sensitive protein alignment using DIAMOND. Nature Methods 2014;12:59-60. 
40. Bushnell B. BBMap: A Fast, Accurate, Splice-Aware Aligner.

41. Gerlt JA, Bouvier JT, Davidson DB, Imker HJ, Sadkhin B, et al. Enzyme function initiativeenzyme similarity tool (EFI-EST): a web tool for generating protein sequence similarity networks. Biochimica Et Biophysica Acta (BBA)-Proteins and Proteomics 2015;1854:10191037.

42. Shannon P, Markiel A, Ozier O, Baliga NS, Wang JT, et al. Cytoscape: a software environment for integrated models of biomolecular interaction networks. Genome research 2003;13:2498-2504.

43. Katoh K, Standley DM. MAFFT multiple sequence alignment software version 7: improvements in performance and usability. Molecular biology and evolution 2013;30:772780.

44. Guindon S, Dufayard J-F, Lefort V, Anisimova M, Hordijk W, et al. New algorithms and methods to estimate maximum-likelihood phylogenies: assessing the performance of PhyML 3.0. Systematic biology 2010;59:307-321.

45. Rambaut A, Drummond A. FigTree: Tree figure drawing tool, version 1.2. 2. Institute of Evolutionary Biology, University of Edinburgh.

46. Paraskevopoulou S, Käfer S, Zirkel F, Donath A, Petersen M, et al. Viromics of extant insect orders unveil the evolution of the flavi-like superfamily. Virus Evolution 2021;7:veab030.

47. Culley AI, Lang AS, Suttle CA. High diversity of unknown picorna-like viruses in the sea. Nature 2003;424:1054-1057.

48. Zhang $Y-Y$, Chen $Y$, Wei $X$, Cui J. Viromes in marine ecosystems reveal remarkable invertebrate RNA virus diversity. Sci China Life Sci. Epub ahead of print 17 June 2021. DOI: 10.1007/s11427-020-1936-2.

49. Lang AS, Vlok M, Culley AI, Suttle CA, Takao Y, et al. ICTV Virus Taxonomy Profile: Marnaviridae 2021. Journal of General Virology 2021;102:001633.

50. Brussaard CPD, Noordeloos AAM, Sandaa R-A, Heldal M, Bratbak G. Discovery of a dsRNA virus infecting the marine photosynthetic protist Micromonas pusilla. Virology 2004;319:280-291.

51. Day JM. The diversity of the orthoreoviruses: molecular taxonomy and phylogentic divides. Infection, Genetics and Evolution 2009;9:390-400.

52. Valles SM, Chen Y, Firth AE, Guérin DMA, Hashimoto Y, et al. ICTV virus taxonomy profile: Iflaviridae. The Journal of General Virology 2017;98:527. 
53. Guo L, Lu X, Liu X, Li P, Wu J, et al. Metatranscriptomic Analysis Reveals the Virome and Viral Genomic Evolution of Medically Important Mites. Journal of Virology;95:e01686-20.

54. Steil BP, Kempf BJ, Barton DJ. Poly(A) at the 3] End of Positive-Strand RNA and VPg-Linked Poly $(U)$ at the 5? End of Negative-Strand RNA Are Reciprocal Templates during Replication of Poliovirus RNA. Journal of Virology 2010;84:2843-2858.

55. Gann ER, Kang Y, Dyhrman ST, Gobler CJ, Wilhelm SW. Metatranscriptome Library Preparation Influences Analyses of Viral Community Activity During a Brown Tide Bloom. Frontiers in Microbiology 2021;12:1126.

56. Canuti $\mathbf{M}$, van der Hoek L. Virus discovery: are we scientists or genome collectors? Trends in microbiology 2014;22:229-231. 
434 Figure 1: Summary of viral contigs discovered from echinoderm transcriptomes (A) The number of transcriptomes downloaded from NCBI ordered by echinoderm class that were processed for viral discovery. (B) Top bars display the total number of viral contigs discovered separated by echinoderm class and life stage. Bottom bars display total number of transcriptomes separated by echinoderm class and life stage (C) Percentage of viral reads in transcriptomes by
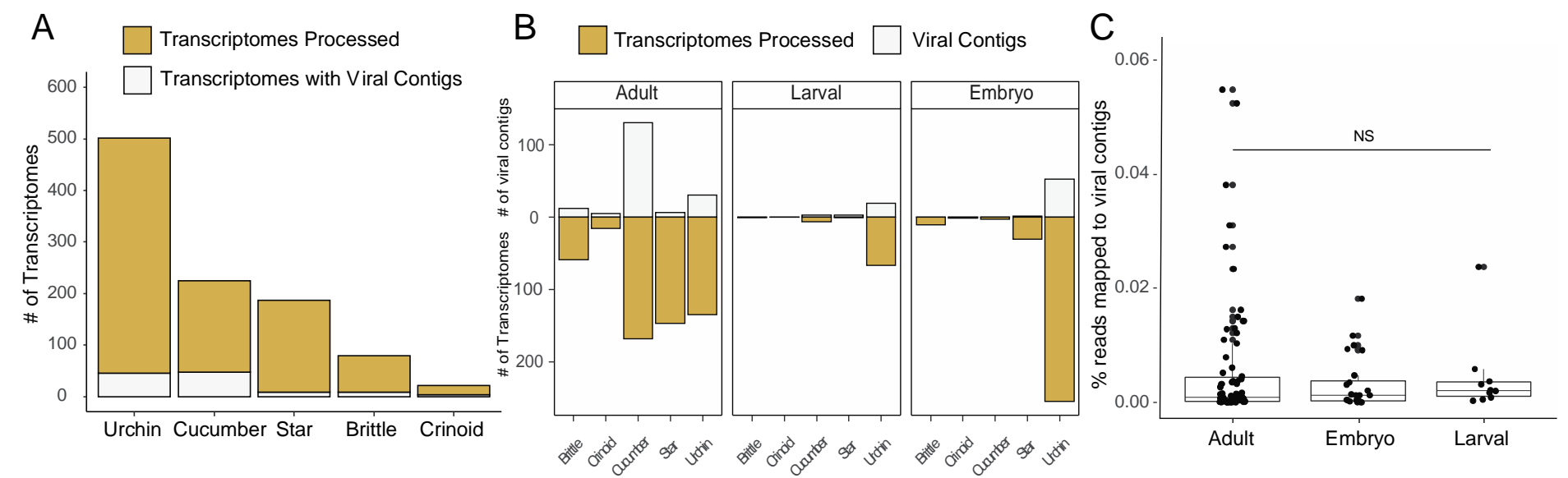
Figure 2: Picornaviruses are the dominant viral order found in echinoderms. (A) Colored circles represent viral sequences discovered from echinoderm transcriptomes and RNA viral

470 metagenomes. White circles are viral genomes taken from NCBI. (B) The bar chart displays the

A

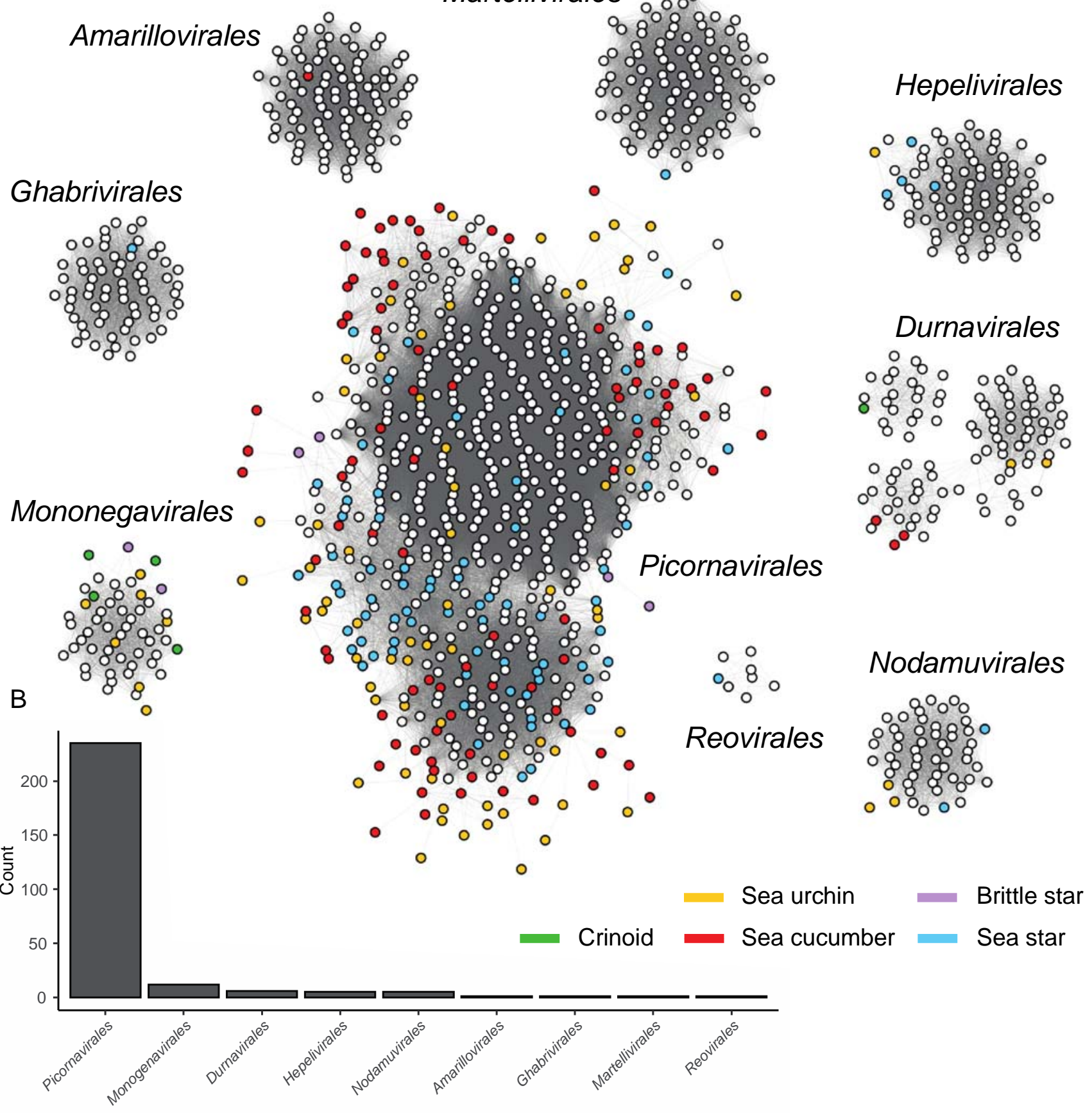


bioRxiv preprint doi: https://doi.org/10.1101/2022.03.01.482561; this version posted March 2, 2022. The copyright holder for this preprint (which was not certified by peer review) is the author/funder, who has granted bioRxiv a license to display the preprint in perpetuity. It is made available under aCC-BY-ND 4.0 International license.

476 Figure 3: Echinoderm picornaviruses are broadly distributed across the Picornavirales

477 phylogeny. Tips are colored by taxonomic family with orange circles representing echinoderm 478 picornaviruses. Genome architectures of complete and near complete genomes recovered from 479 assemblies displayed. Genomes are drawn approximate to scale in a 5' to 3' direction. Open 480 reading frames denoted by boxes and colored by general function. Asterisk represents an 481 incomplete open reading frame. Animal icons represent the echinoderm order the viral contig is 482 associated with.

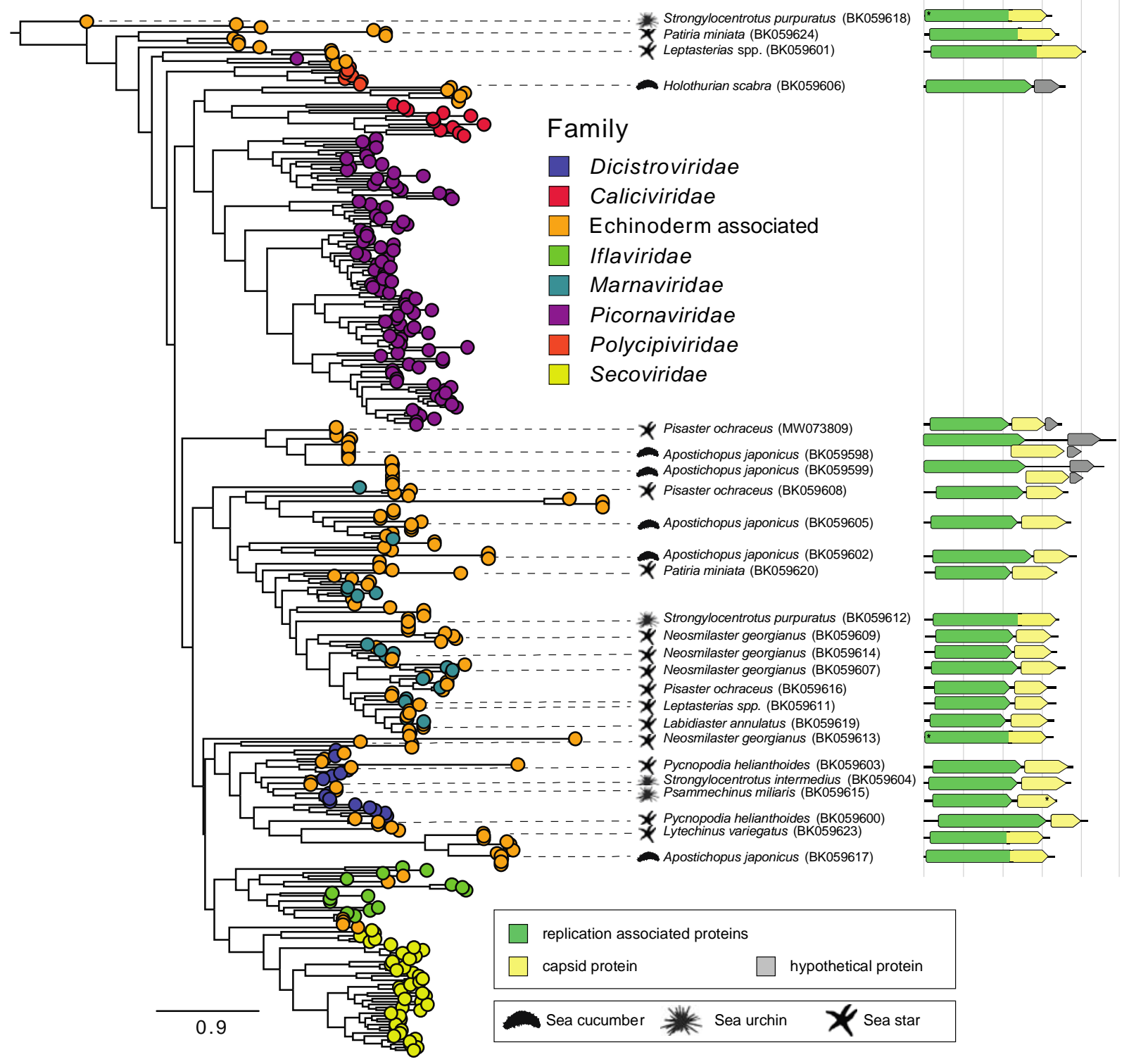


bioRxiv preprint doi: https://doi.org/10.1101/2022.03.01.482561; this version posted March 2, 2022. The copyright holder for this preprint (which was not certified by peer review) is the author/funder, who has granted bioRxiv a license to display the preprint in perpetuity. It is made available under aCC-BY-ND 4.0 International license.

Figure 4: Phylogenetic placement of echinoderm viruses from Reovirales, Mononegavirales, Amarillovirales, and Hepevirales. Tips are colored by taxonomic family or genus with black circles representing echinoderm viruses. Genome architectures of complete and near complete genomes recovered from assemblies displayed. Genomes are drawn approximate to scale in a 5' to 3' direction. Open reading frames denoted by boxes and colored by general function. Asterisk represents an incomplete open reading frame and a question mark denotes potentially incomplete
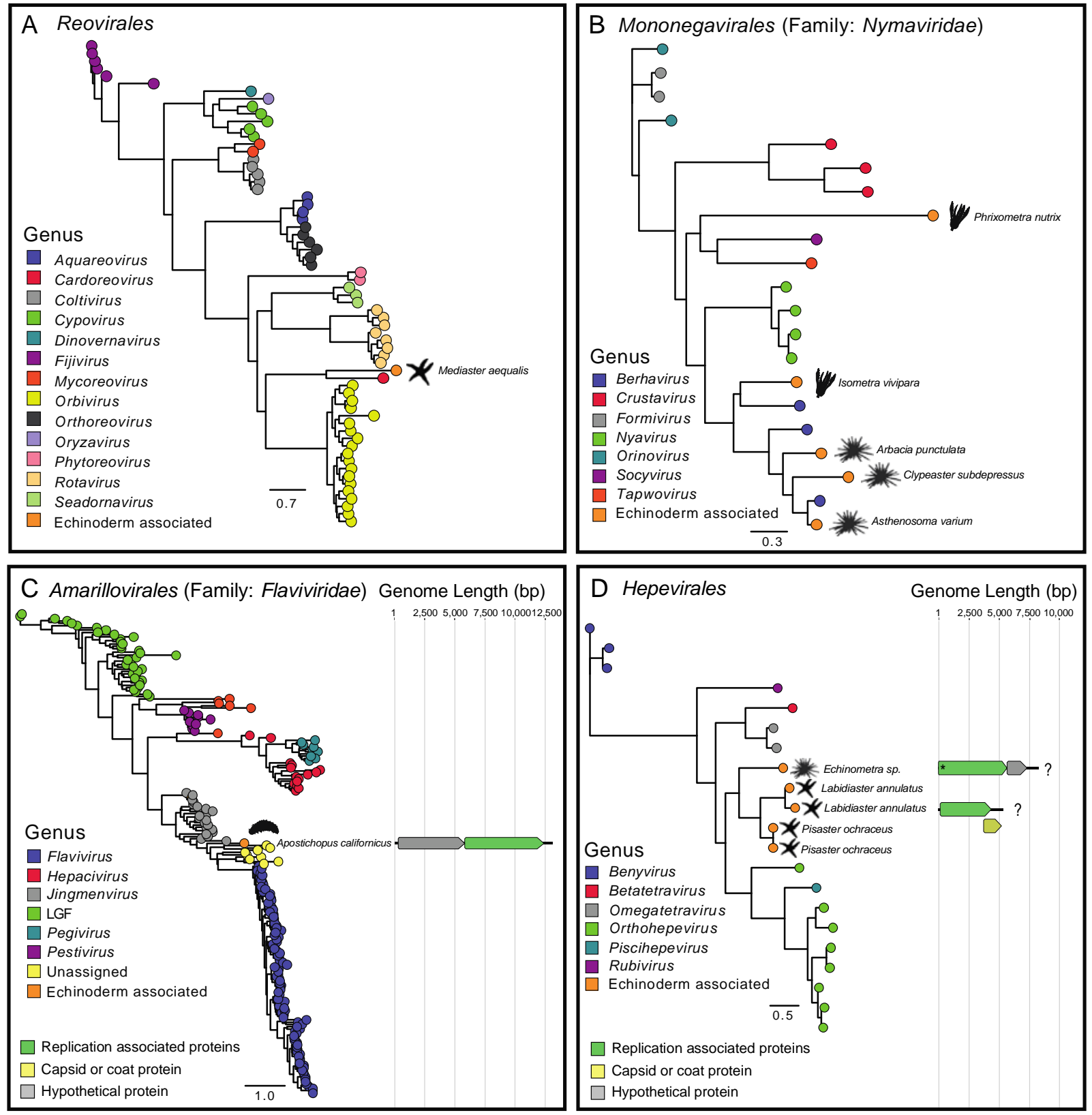

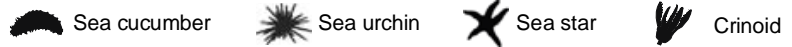


bioRxiv preprint doi: https://doi.org/10.1101/2022.03.01.482561; this version posted March 2, 2022. The copyright holder for this preprint (which was not certified by peer review) is the author/funder, who has granted bioRxiv a license to display the preprint in perpetuity. It is made available under aCC-BY-ND 4.0 International license.

Figure 5: Genome architectures and comparison of complete or near complete genomes recovered from assemblies. Genomes are drawn approximate to scale in a 5' to 3' direction. Open reading frames denoted by boxes and colored regions represent conserved protein domains. Asterisk represents an incomplete open reading frame and question marks indicate missing open reading frames that would complete the genome. Animal icons represent the echinoderm order the viral contig is associated with.

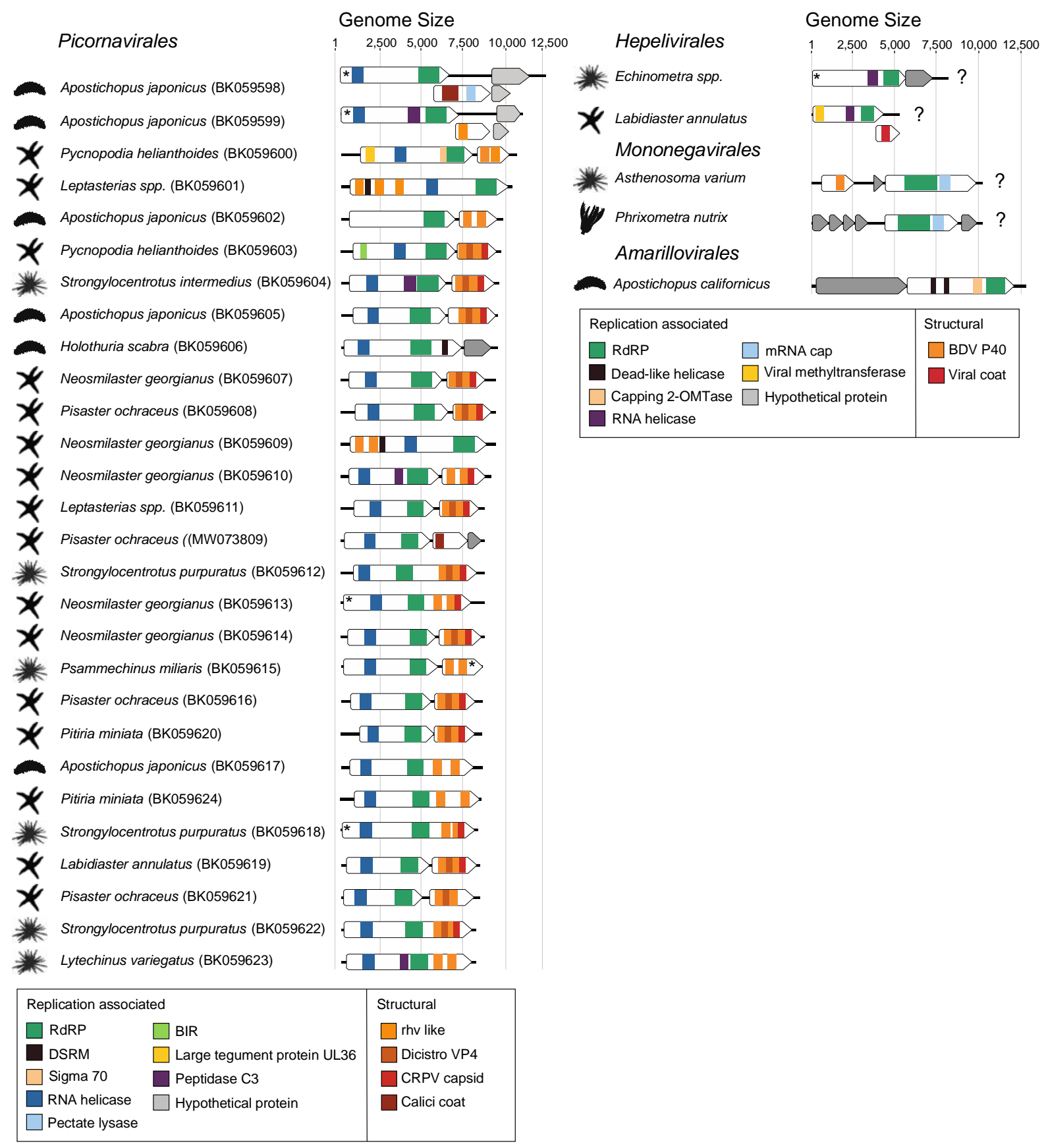


Figure S1: Summary statistics of viral discovery from short read libraries. (A) The

502 Total number of viral contigs discovered in RNA viral metagenomes and host transcriptomes.

503 (C) Pearson's correlations of total reads in a given library and total viral sequences in the same

504 library.

505

506
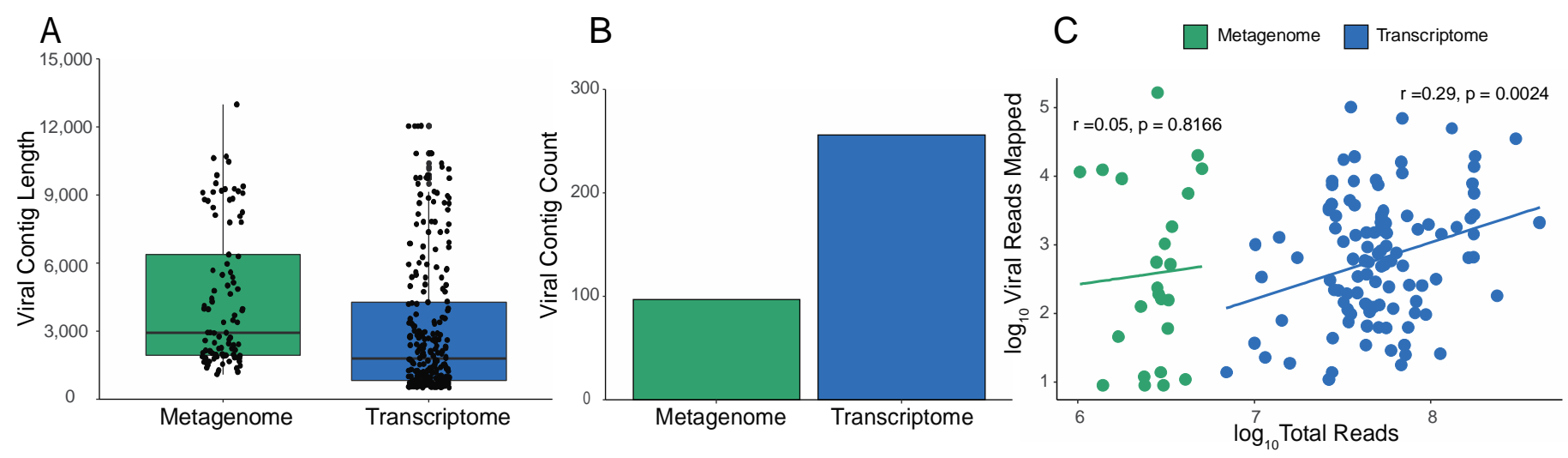